\title{
Parameters affecting water hammer in metal pipelines
}

\author{
Kamil Urbanowicz ${ }^{1, *}$, and Mateusz Firkowski ${ }^{1}$ \\ ${ }^{1}$ West Pomeranian University of Technology Szczecin, Department of Mechanical Engineering and \\ Mechatronics, Piastów 19, 70-310 Szczecin, Poland
}

\begin{abstract}
The water hammer related to rapid wave pressure changes in hydraulic systems have been subjected to intensive research for more than a hundred years. Nevertheless, a large number of new papers appear each year. Current literature indicates model differences resulting from the used material of the pipe. In the hydraulic machinery, elastic (metal) pipes are usually used, while water transport in water supply system is currently realized with pipes whose deformation of the walls is viscoelastic. In this paper, the individual and group impact of all parameters influencing the results of numerical modelling of the water hammer occurring in the pipes will be analysed. The method of characteristics will be used to solve partial differential equations describing the flow.
\end{abstract}

\section{Introduction}

In high-pressure hydraulic systems, central heating, cooling of cores in nuclear power plants as well as in long transmission lines, pipes made of metals are still widely used. In them, the pressure wave propagation velocity are approximately 3-4 times higher than in plastic pipes. Therefore, also the pressure increase taking place during the water hammer, which is calculated from the Joukowsky's formula:

$$
\Delta p=c \rho \Delta v,
$$

exceeds at least three times the increase that would occur in an analogue plastic conduit. In addition, the deformation of the pipe walls is elastic, and not as in the case of plastic pipes, viscoelastic [1], the maximum possible pressures may even double the value calculated from formula (1). Such an increase may be the result of the superposition of pressure waves (primary and secondary) during the flows in which liquid column separation occur commonly known as cavitation [2,3]. As shown by the latest papers, the vibrations of pipe [4-6] also have great model significance, however due to their complexity they will not be included in this work.

Usually, hydraulic systems are protected against the harmful effects of pressure increases by using specialized damping devices (air tanks, compensating tanks, vents, safety and return valves, etc. [7-9]). In order to avoid high costs of the aforementioned protective devices, one should use (if possible) slow-closing valves, which will not lead to quick water hammers, which are the most serious in consequences. It is also worth to

* Corresponding author: kamil.urbanowicz@zut.edu.pl 
optimize such systems at the design stage through appropriate selection of: parameters characterizing the pipe $\left(L, E_{0}, R, e\right)$, its constraints $(\alpha, \Xi)$ and the working medium $(\rho, K, v)$. Hydraulic impact should not be treated only as a problem, in practice one can try to use the advantages of this phenomenon by improving the work of hydraulic rams [10] and devices generating mechanical vibrations [11]. What is the impact of individual parameters on the flow, will be shown by the results of simulation tests carried out in this work.

\section{Basic equations describing transient flow}

The equation of continuity and motion that represent the transient flow have the form:

$$
\left\{\begin{array}{c}
\frac{\partial p}{\partial t}+\rho c^{2} \frac{\partial v}{\partial x}=0 \\
\rho \frac{\partial v}{\partial t}+\frac{\partial p}{\partial x}+\frac{2 \rho}{R}\left(\frac{v|v|}{8} f+\frac{2 v}{R} \int_{0}^{t} \frac{\partial v}{\partial t}(u) \cdot w(t-u) d u\right)=0
\end{array}\right.
$$

The pressure wave speed $c=f\left(\rho, K, v_{p}, R, J_{0}, e\right)$ is given by:

$$
c=\sqrt{\frac{1}{\rho\left[\frac{1}{K}+\frac{\Xi}{E_{0}}\right]}}
$$

As can be seen from formula (3) in single-phase flow, the pressure wave speed is a function of six parameters. Some of them when modelling water hammer occur only in the pressure wave speed formula, they are $K$ and $E_{0}$. The parameter $\alpha$ is calculated, for thick wall pipes $(D / e)<25$ anchored along its length, by the following formula:

$$
\alpha=\left(1-v_{P}^{2}\right) \frac{D}{D+e}+\left(1+v_{P}\right) \frac{2 e}{D}
$$

Friction factor in laminar flow is a function of kinematic viscosity, velocity and pipe inner radius (Reynolds number):

$$
f(v, v, R)=\frac{64}{R e}=\frac{64 v}{v D}=\frac{32 v}{v R} .
$$

In turbulent flow the friction factor is calculated from implicit Colebrook-White equation and is moreover a function of the average height of surface irregularities $\varepsilon$ :

$$
\frac{1}{\sqrt{f}}=-2 \log \left(\frac{\varepsilon}{7.4 R}+\frac{1.255 v}{v R \sqrt{f}}\right) \text {. }
$$

In this work the most accurate Goudar-Sonad [12] approximation of above implicit equation is used. As there are no analytical solutions of the presented water hammer equations (2), and there are still no ready-made computer applications [13] that would allow for simulation of transient flows with acceptable accuracy. In this work a numerical method of characteristics is applied. Then the solution for the inner grid node can be found as:

$$
\left\{\begin{array}{l}
p_{i, t+\Delta t}=\frac{1}{2}\left[\left(p_{i-1, t}+p_{i+1, t}\right)+c \rho\left(v_{i-1, t}-v_{i+1, t}\right)+\frac{2 c \Delta t}{R}\left(\tau_{i+1, t}-\tau_{i-1, t}\right)\right] \\
v_{i, t+\Delta t}=\frac{1}{2}\left[\left(v_{i-1, t}+v_{i+1, t}\right)+\frac{1}{c \rho}\left(p_{i-1, t}-p_{i+1, t}\right)-\frac{2 \Delta t}{\rho R}\left(\tau_{i+1, t}+\tau_{i-1, t}\right)\right]
\end{array} .\right.
$$

The wall shear stress $\tau$ is calculated from the corrected efficient convolution integral solution [14-16]:

$$
\tau_{i i, t}=\frac{\rho f_{i i, t} v_{i i, t}\left|v_{i i, t}\right|}{8}+\frac{2 \rho v}{R} \sum_{j=1}^{3} \underbrace{\left[A_{j} y_{j, i i, t}+\eta B_{j}\left[v_{i i, t}-v_{i i, t-\Delta t}\right]+[1-\eta] C_{j}\left[v_{i i, t-\Delta t}-v_{i i, t-2 \Delta t}\right]\right]}_{y_{j, i i, t+\Delta t}},
$$

where index $i i$ is $i+1$ or $i-1$ depending on which $\tau$ is calculated, and

$$
A_{j}=e^{-n_{j} \Delta \hat{t}}, B_{j}=\frac{m_{j}}{\Delta \hat{t} n_{j}}\left(1-A_{j}\right), C_{j}=A_{j} B_{j}
$$

The coefficients $m_{i}$ and $n_{i}$ are calculated using analytical solution presented in recent work [17], which were based on the effective calculation algorithm [18]. 


\section{Role of the parameters}

This section is devoted to analysis of the impact of individual parameters occurring in the equations discussed in Section 2 that affect simulated transients. The initial values of the tested parameters (Table 1 - the second column) were adopted using the experimental studies known in the literature conducted in Gdańsk at IMP PAN by Adamkowski and Lewandowski [19]. The analysed experimental setup was composed of two tanks and a copper pipe with a length of $L=98.11 \mathrm{~m}$ connecting them. From the observed experimental dynamic courses of pressure changes, the actual value of the pressure wave speed in the tested system was determined at $c=1311.35 \mathrm{~m} / \mathrm{s}$. For this value, while maintaining the remaining parameters (Table 1 - the second column) and using the numerical division of the pipeline into the selected number $N=16$, very good correspondence of simulated pressure runs was obtained (laminar flow $v_{0}=0.066 \mathrm{~m} / \mathrm{s}$ and turbulent $v_{0}=0.94 \mathrm{~m} / \mathrm{s}$ ) with experimental results, which is confirmed by graphical comparisons from Figure 1 .

Table 1. Initial, minimal and maximal values of water hammer parameters.

\begin{tabular}{|c|c|c|c|c|}
\hline Parameter & $\begin{array}{c}\text { Initial } \\
\text { parameters }\end{array}$ & $\begin{array}{c}\text { Assumed } \\
\text { deviation }\end{array}$ & Minimal value & Maximal value \\
\hline$\rho\left[\mathrm{kg} / \mathrm{m}^{3}\right]$ & 997.65 & $\pm 5 \%$ & $\rho_{1}=948$ & $\rho_{2}=1048$ \\
\hline$R[\mathrm{~m}]$ & 0.008 & $\pm 5 \%$ & $R_{1}=0.0076$ & $R_{2}=0.0084$ \\
\hline$e[\mathrm{~m}]$ & 0.001 & $\pm 5 \%$ & $e_{1}=0.00095$ & $e_{2}=0.00105$ \\
\hline$v_{P}[-]$ & 0.35 & $\pm 10 \%$ & $v_{P_{1}}=0.315$ & $v_{P_{2}}=0.385$ \\
\hline$v\left[\mathrm{~m}^{2} / \mathrm{s}\right]$ & $0.95 \cdot 10^{-6}$ & $\pm 10 \%$ & $v_{1}=0.855 \cdot 10^{-6}$ & $v_{2}=1.045 \cdot 10^{-6}$ \\
\hline$K[\mathrm{~Pa}]$ & $2.2 \cdot 10^{9}$ & $\pm 10 \%$ & $K_{1}=1.98 \cdot 10^{9}$ & $K_{2}=2.42 \cdot 10^{9}$ \\
\hline$E_{0}[\mathrm{~Pa}]$ & $124 \cdot 10^{9}$ & $\pm 10 \%$ & $E_{1}=111.6 \cdot 10^{9}$ & $E_{2}=136.4 \cdot 10^{9}$ \\
\hline
\end{tabular}

a) laminar case

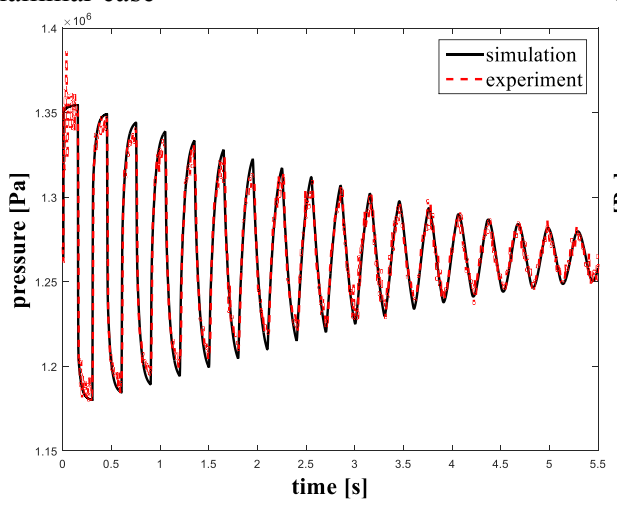

b) turbulent case

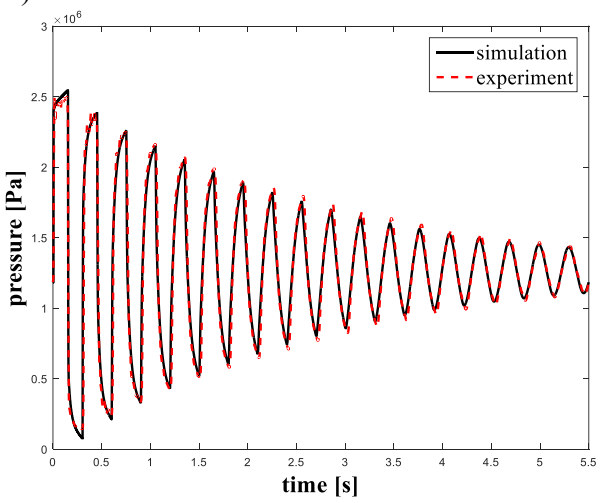

Fig. 1. Fit of simulation results for initial values of tested parameters given in Table 1 .

Often, in the simulation calculations, data regarding hydraulic systems are not accurately taken into account. This is due to the lack of mathematical formulas that would allow determination of the necessary parameters representing the flowing liquid $(\rho, K, v)$ and the pipe material $\left(E_{0}, v_{p}\right)$ as a function of temperature and pressure. In addition, it must not be forgotten that the pipes are made with a certain dimensional tolerance, thus the actual wall thickness $e$ and the internal radius $R$ may differ from those given in the manufacturers' catalogues. To clearly visualize the impact of individual input parameters on the modelled flow, large deviations from initial values have been assumed. Therefore, for $\rho, R$ and $e$ parameters, a possibility of $\pm 5 \%$ deviation of their values was assumed, while for $v, v_{P}, \mathrm{~K}$ 
and $E_{0}$ as large as $\pm 10 \%$ deviation. Accordingly, the appropriate chosen minimum and maximum values of the analysed parameters were calculated and summarized in the fourth and fifth columns of Table 1 .

a) laminar case

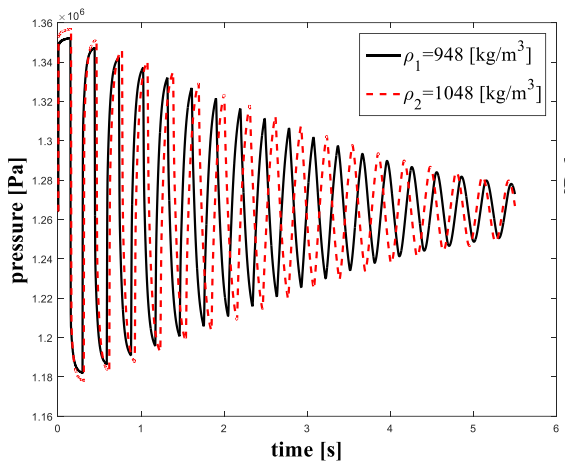

c) laminar flow

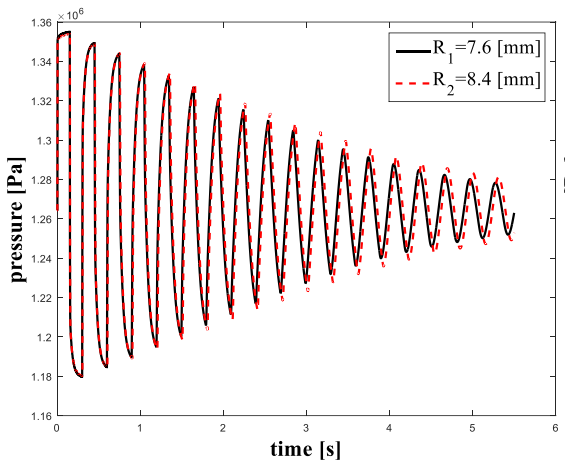

e) laminar flow

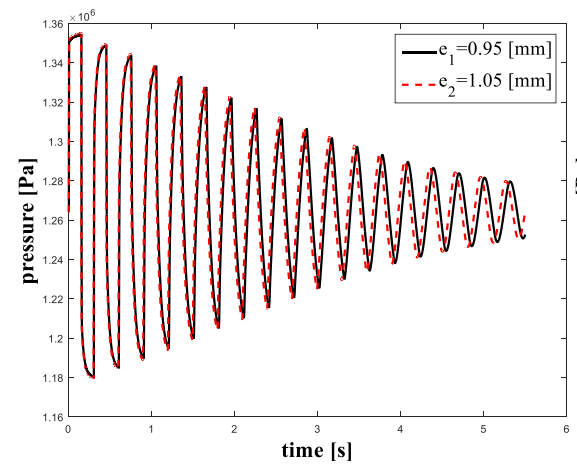

b) turbulent case

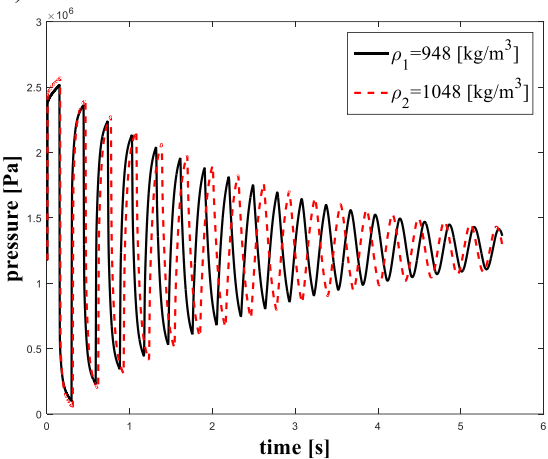

d) turbulent flow

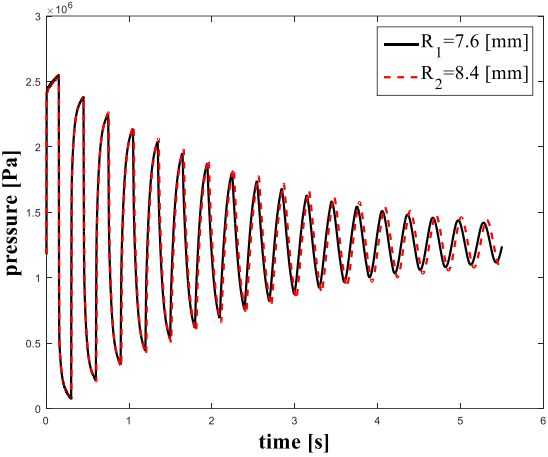

f) turbulent flow

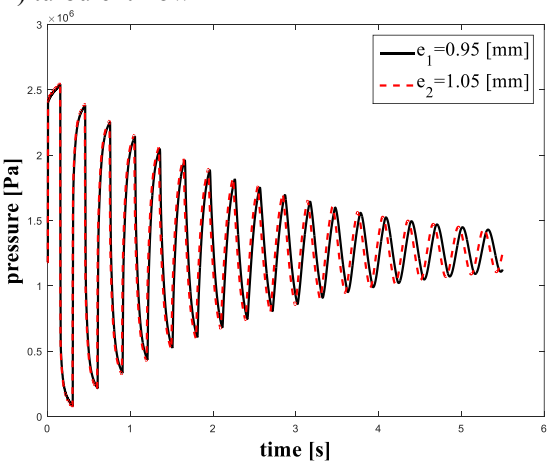

Fig. 2. Liquid density $\rho$, inner pipe radius $R$ and wall thickness $e$ effects (left column: laminar flow results, right column: turbulent flow results).

The results of the numerical simulations carried out, which show the impact of individual parameters, are presented in Figures 2, 3 and 4. From all the analysed parameters only the kinematic viscosity $v$ does not affect the value of pressure wave propagation. The exact influence of the adopted parameters on the values of pressure wave speed $c$ and selected parameters $\left(R, e\right.$, and $\left.v_{P}\right)$ on the reporting support condition of pipe $\alpha$ and on the enhanced $\alpha$ that is denoted by $\Xi$ is shown in Table 2 . 
a) laminar flow

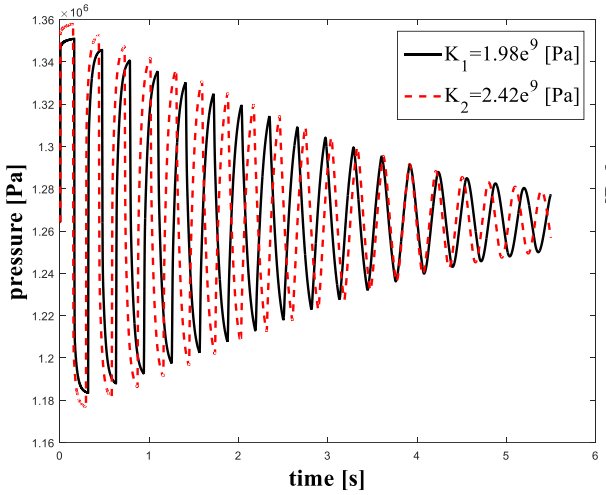

c) laminar flow

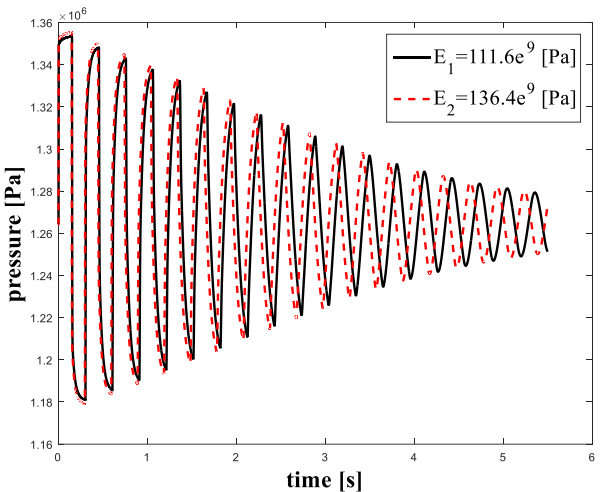

e) laminar flow

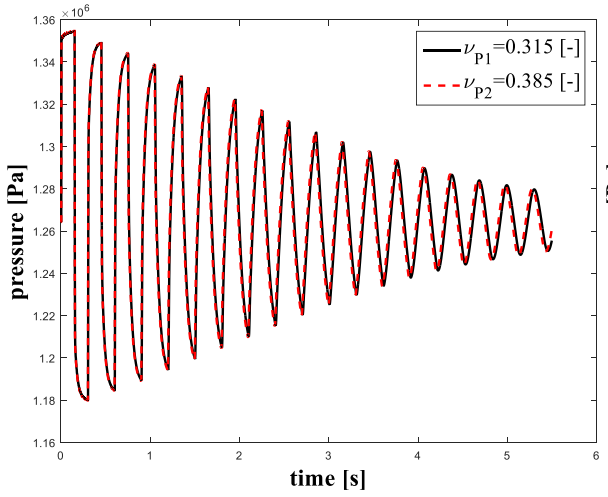

b) turbulent flow

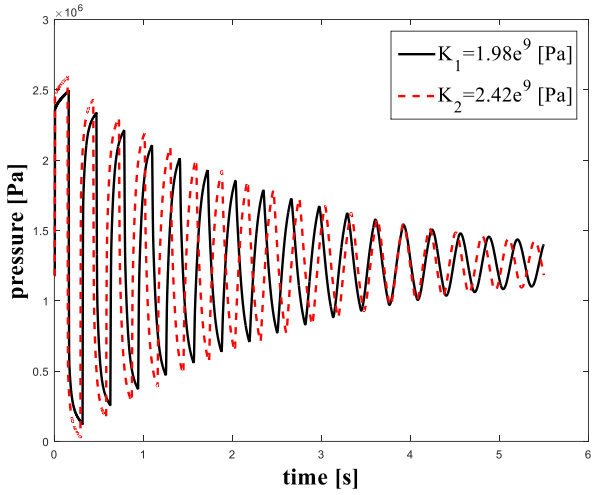

d) turbulent flow

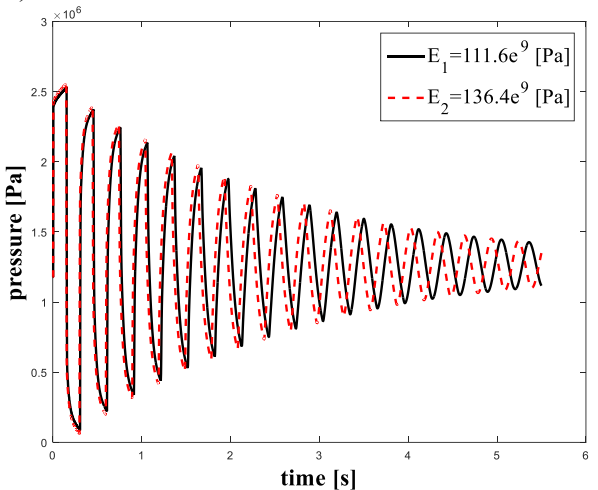

f) turbulent flow

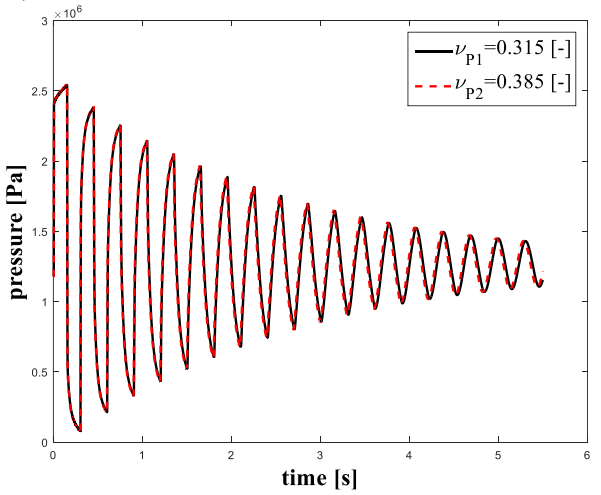

Fig. 3. Liquid bulk modulus $K$, elastic modulus $E_{0}$ and Poisson coefficient $v_{P}$ effects (left colum: laminar flow results, right column: turbulent flow results).

Table 2. Changes of pressure wave speed.

\begin{tabular}{|c|c|c|c|c|c|}
\hline Parameter & $c[\mathrm{~m} / \mathrm{s}]$ & Parameter & $c[\mathrm{~m} / \mathrm{s}]$ & $\Xi[-]$ & $\alpha[-]$ \\
\hline$\rho_{1}$ & 1345.3 & $R_{1}$ & 1317.7 & 15.215 & 1.001 \\
\hline$\rho_{2}$ & 1279.5 & $R_{2}$ & 1305.1 & 16.614 & 0.989 \\
\hline$K_{1}$ & 1258.0 & $e_{1}$ & 1304.7 & 16.651 & 0.987 \\
\hline$K_{2}$ & 1360.5 & $e_{2}$ & 1317.4 & 15.248 & 1.001 \\
\hline$E_{1}$ & 1295.6 & $v_{P_{1}}$ & 1308.8 & 16.195 & 1.012 \\
\hline$E_{2}$ & 1324.7 & $v_{P_{2}}$ & 1314.2 & 15.597 & 0.975 \\
\hline
\end{tabular}


a) laminar flow

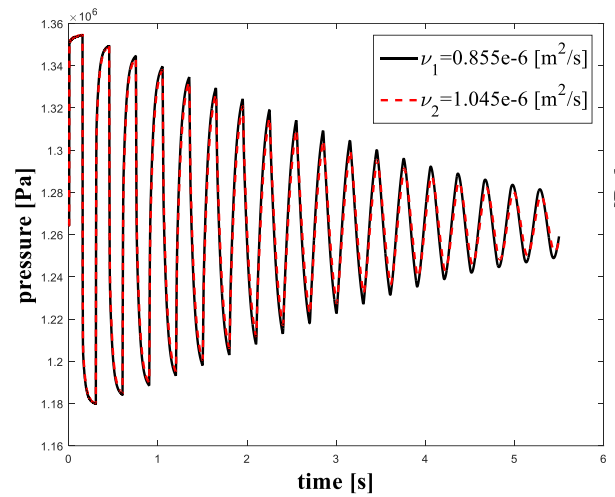

b) turbulent flow

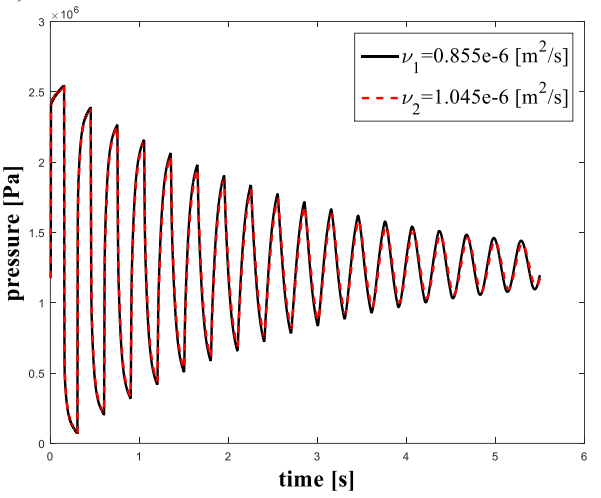

Fig. 4. Liquid kinematic viscosity $v$ effect.

a) laminar flow

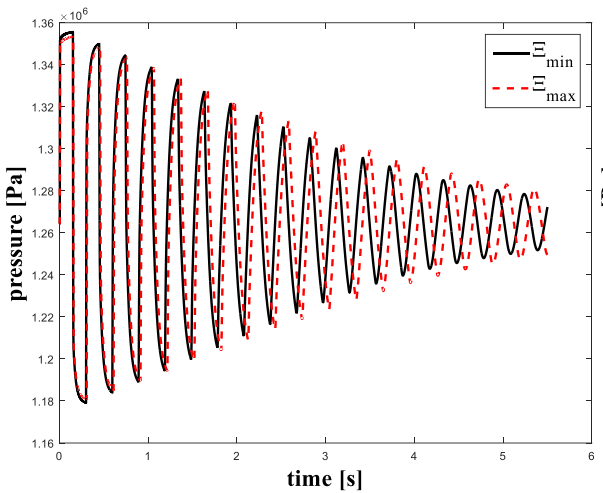

c) laminar flow

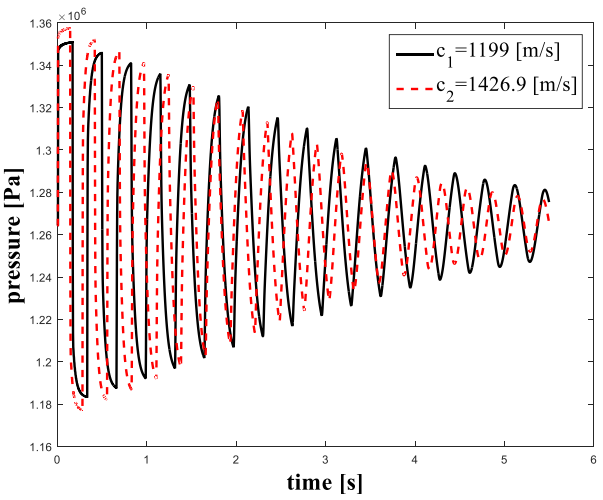

b) turbulent flow

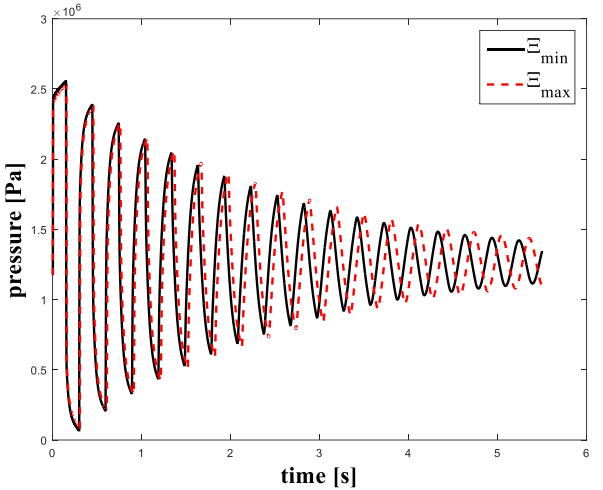

d) turbulent flow

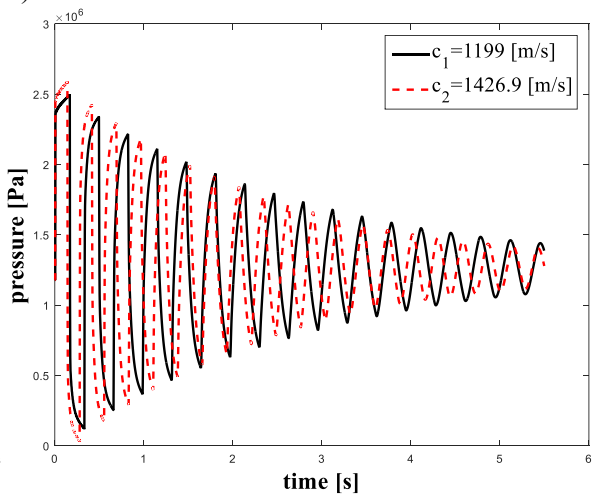

Fig. 5. Multiparameter effects.

Short conclusion resulting from the research for single parameter effects is:

a) For first amplitude $p_{1}$ pressure grow $\uparrow: \rho \uparrow, R \downarrow, e \uparrow, v_{P} \uparrow, K \uparrow, E_{0} \uparrow, v-$ no effect!

b) For eighteen amplitudes $p_{18}$ pressure grow $\uparrow: R \uparrow, v \downarrow$

c) For amplitude delays $T_{18} \uparrow: \rho \uparrow, R \uparrow, e \downarrow, v_{P} \downarrow, K \downarrow, E_{0} \downarrow$.

Complete tendencies is presented in Table 3 . 
Table 3. Single parameter effect.

\begin{tabular}{|c|c|c|c|}
\hline when: $\uparrow$ of & then $p_{1}$ & and $p_{18}$ & and $T_{18}$ \\
\hline$\rho$ & $\uparrow$ & $\rightarrow$ (very small change) & $\uparrow$ \\
\hline$R$ & $\searrow$ & $\uparrow$ & $\nearrow$ \\
\hline$e$ & $\nearrow$ & $\rightarrow$ (very small change) & $\downarrow$ \\
\hline$v_{P}$ & $\nearrow$ & $\rightarrow$ (very small change) & $\searrow$ \\
\hline$v$ & $\rightarrow$ (no change) & $\downarrow$ & $\rightarrow$ (very small change) \\
\hline$K$ & $\uparrow$ & $\rightarrow$ (very small change) & $\downarrow$ \\
\hline$E_{0}$ & $\uparrow$ & $\rightarrow$ (very small change) & $\downarrow$ \\
\hline
\end{tabular}

Multiparameter effect takes places when three parameter changes are assumed. When one assumes: $v_{P_{2}}=0.385, R_{1}=0.0076$ and $e_{2}=0.00105$ then the dimensionless parameters takes values: $\alpha=0.988, \Xi=14.304$ and pressure wave speed is $c=1326.2 \mathrm{~m} / \mathrm{s}$. Otherwise for: $v_{P_{1}}=0.315, R_{2}=0.0084, e_{1}=0.00095$ one get $\alpha=$ $1.001, \Xi=17.707$ and $c=1295.4 \mathrm{~m} / \mathrm{s}$. The differences of the final pressure runs are presented on Fig. 5a and Fig. 5 b.

An extreme case takes place when assuming $\rho_{2}=1048, R_{2}=0.0084, e_{1}=0.00095$, $K_{1}=1.98 \cdot 10^{9}, v_{P_{1}}=0.315, E_{1}=111.6 \cdot 10^{9}$, then the calculated minimal pressure wave speed was: $c_{\min }=1199 \mathrm{~m} / \mathrm{s}$. When assuming $\rho_{1}=948, R_{1}=0.0076$, $e_{2}=0.00105, K_{2}=2.42 \cdot 10^{9}, v_{P_{2}}=0.385, E_{2}=136.4 \cdot 10^{9}$, the calculated pressure wave speeds was $c_{\max }=1426.9 \mathrm{~m} / \mathrm{s}$. The difference of the pressure wave speed between two extreme cases was $228 \mathrm{~m} / \mathrm{s}$. For $c_{\min }$ the number of pressure amplitudes that was simulated in analysed 5.5 seconds from quick step closing of valve was seventeen, and for $c_{\text {max }}$ the number of simulated amplitudes was twenty (Fig. 5c, Fig. 5d).

The obtained test results showed that the temporal compliance of the modelled amplitudes depends strictly on the value of the calculated pressure wave speed. Six parameters assumed before calculations start, affect this speed. Two of them are related to liquid properties: $K$ - bulk modulus, $\rho$ - liquid density. The four remaining ones are related to the properties of the pipe in which the unsteady flow is analysed: $E_{0}-$ Young's modulus and $v_{P}$ - Poisson's ratio (material constants); $R$ - pipe inner radius and $e$ - pipe wall thickness. From the analysis of pressure values on the simulated eighteenth amplitude, it follows that the change in the velocity of pressure wave speed does not have so much significance as the change in the viscosity coefficient $v$ of the flowing liquid. This is due to the fact that the coefficient of kinematic viscosity occurs only in the mathematical expressions of the final formulas that are responsible for modelling the impact of the hydraulic resistance of the flowing liquid.

\section{Conclusions}

Investigations carried out in this work allowed to determine typical tendencies occurring during water hammer in metal pipes in which there is no additional cavitation disruption of the flow. Knowledge of individual and group impact of selected parameters will allow better control in the wider perspective and thus limit the negative impact of the analysed phenomenon. The presented studies also showed what error can be made as a result of the rough assumption of some parameters. This can lead to accumulation of errors and the analysed flow will be modelled with a large error. To avoid this, one should always enter data about flowing fluid and system with high accuracy in the design and testing of new and existing systems. It would be good to collect the latest formulas for the physical properties of selected liquids used in water and hydraulic systems $(K, \rho, v)$ as well as 
equations for the influence of temperature and pressure inside the pipe on the material properties of the pipe $\left(E_{0}, v_{P}\right)$. In addition, the actual internal diameters and pipe wall thicknesses should always be checked as the producers always carry them within the scope of a certain manufacturing tolerance. Soon similar tests will be performed for cavitation flows so that the effect of the assumed vapor pressure may also be determined.

\section{List of symbols and units}

$c$-pressure wave speed $[\mathrm{m} / \mathrm{s}], e$-thickness of pipe wall $[\mathrm{m}], E_{0}$ - pipe elastic modulus $[P a], f-$ friction coefficient $[-], K$ - liquid bulk modulus $[P a], p-$ pressure $[P a]$, $R-$ inner pipe radius $[m], t-$ time $[s], v-$ velocity $[m / s], w(t-u)-$ weighting function $[-], x$-distance along the pipe $[m], \alpha$-parameter describing support condition of pipe $[-], \Delta v-$ velocity change $[\mathrm{m} / \mathrm{s}] ; v-$ kinematic viscosity $\left[\mathrm{m}^{2} / \mathrm{s}\right], \quad \rho-$ liquid density $\left[\mathrm{kg} / \mathrm{m}^{3}\right], \Xi=2 \frac{R}{e} \alpha-$ enhanced $\alpha$ parameter $[-]$

\section{References}

1. K. Urbanowicz, M. Firkowski, Z. Zarzycki, J. Phys.: Conf. Ser., 760(1), 012037 (2016)

2. A. Bergant, A.R. Simpson, A.S. Tijsseling, J. Fluids Struct., 22(2), 135-171 (2006)

3. Z. Zarzycki, K. Urbanowicz, Chem. Process Eng., 27(3), 915-933 (2006)

4. S. Henclik, J. Sound Vib., 417, 245-259 (2018)

5. S. Henclik, J. Fluids Struct., 76, 469-487 (2018)

6. A.S. Tijsseling, Comput. Struct., 85(11), 844-851 (2007)

7. A. Rogalev, A. Kocherova, I. Komarov, I. Garanin, G. Kurdiukova, Contemp. Eng. Sci., 8(23), 1067-1081 (2015)

8. D.D. Li, J. Jiang, Z. Zhao, W.S. Yi, G. Lan, IOP Conf. Ser.: Mater. Sci. Eng., 52(1), 072007 (2013)

9. Y. Wu, Y. Xu, C. Wang, Procedia Engineer., 119(1), 884-891 (2015)

10. W. Sobieski, D. Grygo, S. Lipiński, Sadhana-Acad. P. Eng. S., 41(11), 1333-1347 (2016)

11. K. Suzuki, K. Yokoyama, Y. Endo, Y Shibata, Proc. $6^{\text {th }}$ JFPS Int. Symp. Fluid Power, 41-46 (2005)

12. C.T. Goudar, J.R. Sonnad, Hydrocarb. Process., 87(8), 79-83 (2008)

13. A. Niedźwiedzka, S. Lipiński, S. Kornet, J. Hydroinform., 19(5), 653-665 (2017)

14. K. Urbanowicz, Proc. $12^{\text {th }}$ Int. Conf. Press. Surges, 113-130 (2015)

15. K. Urbanowicz, Z. Zarzycki, J. Theor. App. Mech.-Pol., 53(2), 295-305 (2015)

16. K. Urbanowicz, Pol. Marit. Res., 24(3), 68-77 (2017)

17. K. Urbanowicz, J. Theor. App. Mech.-Pol., 55(3), 1029-1040 (2017)

18. K. Urbanowicz, Proc. $11^{\text {th }}$ Int. Conf. Press. Surges, 477-492 (2012)

19. A. Adamkowski, M. Lewandowski, J. Fluids Eng., 128, 1351-1363 (2006) 